\title{
GRÉGARINES NOUVELLES \\ DE COLÉOPTÈRES TÉNÉBRIONIDES
}

\section{Par Odette TUZET et Jean THÉODORIDÉS}

Dans l'intestin moyen de quelques Ténébrionides, récoltés aux environs de Banyuls-sur-Mer (Pyrénées-Orientales) (garrigues voisines du chemin de la Tour Madeloc, plage d'Argelès-sur-Mer, Dunes du Canet), en Corse (environs du Cap Corse), au Maroc (Casablanca), et en Mauritanie (Atar), nous avons pu observer quelques espèces nouvelles de Grégarines Polycystidées, que nous allons décrire ici.

Les Coléoptères Ténébrionides étudiés sont :

1. Tentyria mucronata Stev.

2. Asida sericea $\mathrm{Ol}$.

3. Akis punctata Thunb.

4. Akis elegans Sol.

5. Pimelia angulata F. ssp. angulosa Ol.

Pour plus de commodité les Grégarines seront classées par hòtes:

\section{1. - TENTYRIA MUCRONATA Stev.}

Dans l'intestin moyen d'adultes de cette espèce provenant de la plage d'Argeìès-sur-Mer (X. 1949, VI. 1950) et des dunes du Canet (V. et VII. 1950) (Pyrénées-Orientales), nous avons trouvé deux Grégarines appartenant l'une au genre Stylocephalus et l'autre au genre Sphrrocystis.

Les Stylocephalus ne se montrent pas toujours sous des aspects semblables et nous avons cru, tout d'abord, avoir affaire à deux espèces différentes. Certaines formes sont lisses, d'autres annelées, mais nous avons vu des intermédiaires entre ces deux formes extrêmes qui nous ont montré que nous avions affaire à une même espèce à laquelle nous donnerons, à cause de cette particularité, le nom de :

Ann. de Parasitologie, T. XXVI, $\mathrm{x}^{\circ}$ 5-6. -1951. 


\section{Stylocephalus variabilis n. sp.}

Ce Stylocephalus diffère de S. oblongatas Hamm. et de $S$. longicollis Sten. par l'extrémité de son épimérite qui n’est pas trifoliée comme celui de ces deux espèces, mais sphérique ; de plus, la segmentation du deutomérite n'a jamais été signalée chez ces deux Grégarines.
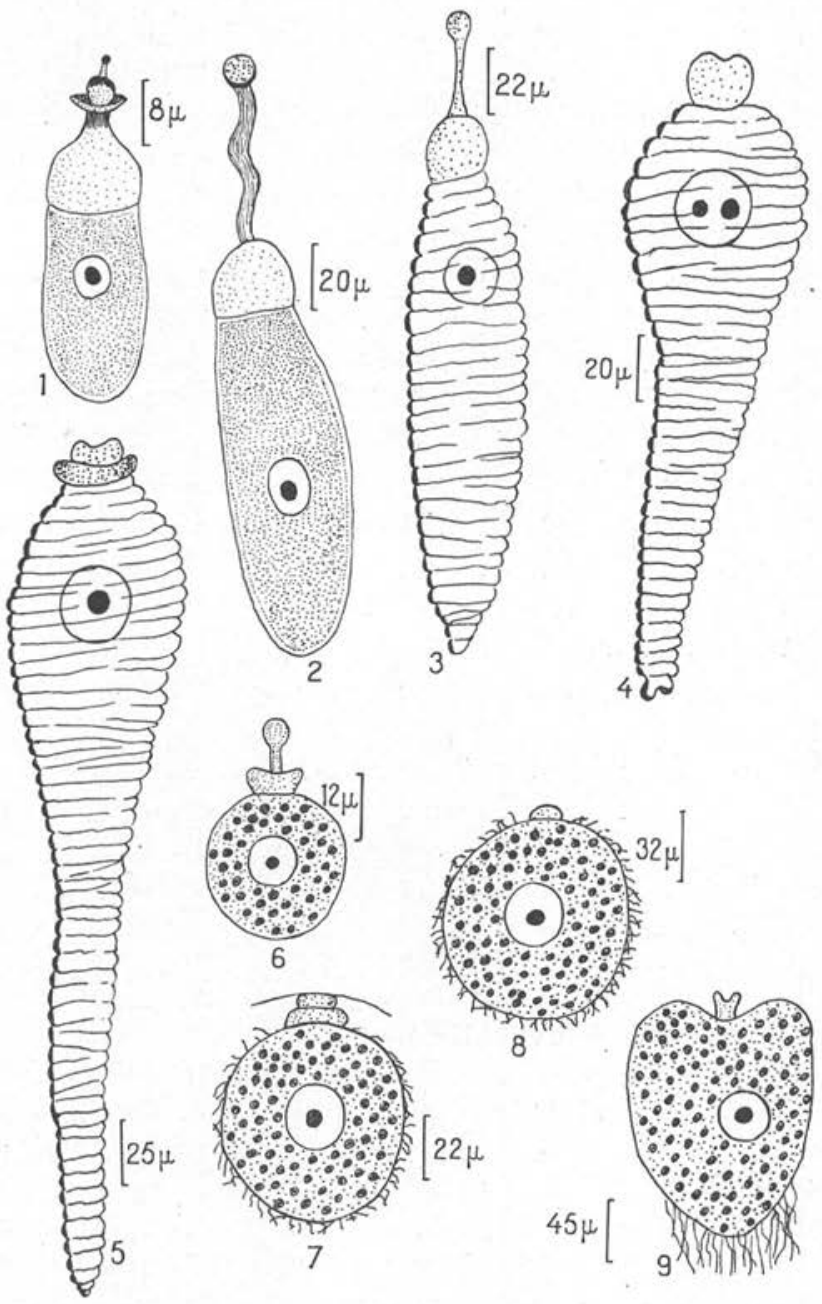

Fig. I. - Grégarines de Tentyria mucronala Stev. 1-2-3-4-5, Styloccphalus variabilis n. sp. (1-2: formes lisses ; 3-4-5 : formes plissées), 6- $3-8-9$, Sphærocystis tentyrize n. sp. 
Les formes jeunes de $S$. variabilis ont un épimérite dont le col ne s'est pas encore développé (fig. I, 1) ; il montre antérieurement un granule auquel fait suite un court prolongement venant s'insérer sur une sphérule. La base de cette sphérule adhère au fond d'une cupule représentant la partie antérieure du protomérite. Celuici est bien développé et a environ le tiers de la longueur du deutomérite. Ces jeunes Grégarines sont lisses.

Puis, l'épimérite se différencie : il est fait d'un long col portant un renflement antérieur sphérique (fig. I, 2) ; le protoplasme de la sphérule est très granuleux et le col a souvent l'aspect fibrillaire. Ces formes à épimérite bien développé peuvent être soit lisses (fig. I, 2), soit annelées (fig. I, 3). Dans ce cas, le deutomérite est complètement plissé ou l'est en partie seulement, et c'est alors, le plus souvent, la partie postérieure qui l'est.

Pour nous, cette segmentation du deutomérite est superficielle ; elle n'intéresse pas le protoplasme et est dùe à des plis circulaires d'une épaisse membrane. Le noyau logé dans le deutomérite est sphérique ou ovoïde avec un nucléole.

Puis, par perte de son épimérite, la Grégarine devient libre òns la lumière intestinale ; elle s'allonge et prend alors la forme d'un têtard (fig. I, 4-5). La plupart de ces Stylocephalus sans épimérite sent segmentés sur toute leur longueur. Certains peuvent avoir une partie du deutomérite lisse, mais chez Tentyria mucronata nous n'avons pas observé de grosses Grégarines complètement lisses. Les plus grandes formes que nous ayons vues mesuraient environ $350 \mu$ de long.

Les Tentyria de la plage d'Argelès n'hébergeaient que des $S$. variabilis (1), par contre ceux récoltés dans les dunes du Canet nous ont montré, mélangés au Stylocephalus, des individus d'une Grégarine appartenant à une nouvelle espèce du genre Sphærocystis que nous nommerons :

\section{Sphærocystis tentyriæ n. sp.}

A première vue, il semble que l'on ait affaire à une Monocystidée. Seul, un petit mamelon, souvent invisible, représente le protomérite (fig. I, 8) ; il existe aussi des formes en cœur (fig. I, 9) ; Sphærocystis simplex, décrit par Léger (1892) des larves de Cyphon palli-

(1) Depuis la rédaction de eette note, nous avons eu l'occasion de retrouver Siylocephalus variabilis $\mathrm{n}$. sp. chez des Tent!liria mucronata provenant de Palavas (Hérault) (VII-1951), que nous devons à l'obligeance au professeur H. Harant. 
dulus Boh. (Coléoptère Hélodide) du Poitou, montre aussi des formes plus ou moins allongẻes.

Les jeunes individus (fig. I, 6) ont un deutomérite parfaitement sphérique avec de gros granules de paraglycogène, mais l'épimérite et le protomérite sont bien développés; ils régressent ensuite (fig. I, 7) pour finir par disparaître presque complètement.

Autour de la Grégarine, ou à sa partie postérieure, s'observe souvent une sorte de feutrage fait de fins filaments, dont nous n'avons pu préciser la nature. Il est peut-être fait de fins prolongements émanant de la Grégarine, comme c'est le cas chez plusieurs Monocystidées, ou bien est-il dû à la coagulation d'une sorte de mucus sécrété par celle-ci ?

Ce Sphærocystis différant par plusieurs caractères de $S$. simplex de Léger et de $S$. hydrophili décrit par Foerster (1939a) chez Hydrophilus caraboüdes L., nous croyons bon d'en faire une espèce nouvelle.

\section{2. - ASIDA SERICEA Ol.}

Des exemplaires adultes de cette espèce récoltés à Banyuls-surMer, en mars 1950, dans les garrigues voisines du chemin de la Tour Madeloc, nous ont montré, dans l'intestin moyen deux espèces de Grégarines :

Stylocephalus variabilis, que nous venons de décrire ci-dessus chez Tentyria mucronata, et une espèce nouvelle appartenant au genre Hirmocystis.

Plusieurs Grégarines ont déjà été décrites chez des Asida (pour le détail ef. Watson, 1916) ; ce sont: Stylocephalus giganteus Ellis, chez Asida opaca Say, Asida sp., Eusattus sp. et Eleodes sp. du Colorado (U.S.A), S. oblongatus Hamm. chez Asida grisea (F.) (trouvé également chez Opatrum scibulosum L.), de France, et enfin Hirmocystis asidæ Léger chez Asida servillei Sol. de la province d'Oran (Algérie).

A. Stylocephalus variabilis se présente chez cet hôte avec les mêmes caractères que chez Tentyria mucronata, mais ici, nous avons observé, outre de grandes formes en partie plissées, de grosses Grégarines complètement lisses; ces dernières ressemblent beaucoup à $S$. oblongatus, et nous les avions d'abord considérées comme telles; mais nous n'avons pu observer les stades jeunes des formes lisses et voir l'épimérite caractéristique de $S$. oblongatus. I] existe par contre de nombreuses formes de passage à la fois lisses e! annelées ; c'est pourqtioi il nous semble plus plausible de rattacher ies formes lisses à $S$. variabilis qu'à $S$. oblongatus.

Ann. de Parasitologie, T. XXVI, so 5-6. - 1951. 
Les formes lisses de $S$. variabilis atteignent une longueur de plus d'un millimètre, longueur que l'on ne trouve jamais chez les formes

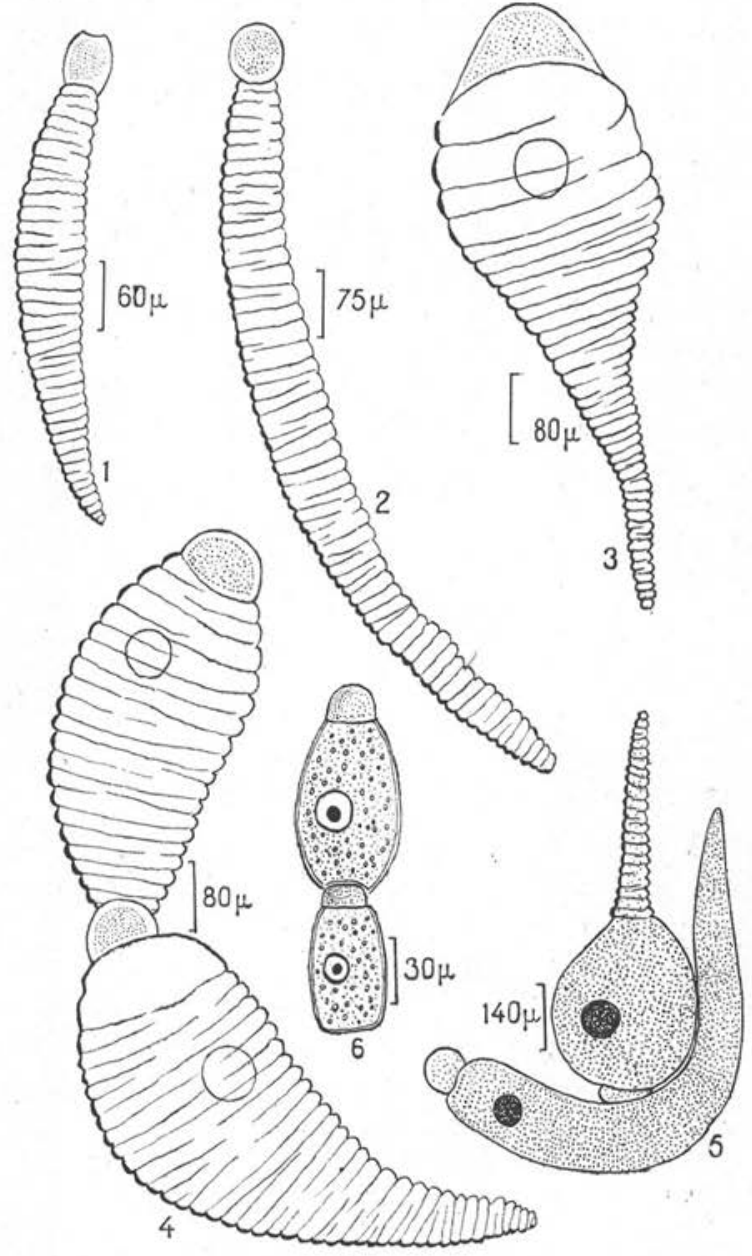

Fıg. II. - Grégarines de Asida sericea Ol. 1-2-3, Stụiocephalus variabilis n. sp. 4-5, Modes d'association des sporadins de $S$. variabilis. 6, Hirmocystis inæqualis n. sp.

plissées les plus développées (fig. II, 2), mais cela se conçoit facilement, les plis prenant sur la longueur du corps.

Comme chez les Tentyria. S. variabilis montre chez A. sericea des formes en têtards (fig. II, 3). 
L'association entre les sporadins de cette espèce nous paraìt digne d'attention. En effet, nous avons pu observer dans nos préparations différents modes d'union de ceux-ci : soit une union latérale où le corps d'un des conjoints présente une concavité dans laquelle se loge l'autre (fig. II, 5), soit une union par les extrémités dissemblables dans laquelle le protomérite d'un des conjoints est fixé au deutomérite de l'autre (fig. II, 4).

Nous n'avons pu observer de couple de gamontes (« coppia gamontica 》) où les deux individus sont réunis par leurs protomérites, comme l'a montré Filipponi (1949) chez Stylocephalus gigas Filip. et $S$. reticulatus Filip. de Blaps gigas L., cette forme d'union devant représenter la syzygie.

Ce dernier auteur (loc. cit.) rappelle d'ailleurs les diverses modalités de l'union des gamontes chez les Polycystidées, d'où résulte une terminologie hétérogène suivant les différents auteurs, et qui, bien souvent prête à confusion. Nous n'insisterons pas ici sur cette question que l'un de nous (J. T.) développera dans un travail ultérieur.

En ce qui concerne $S$. variabilis, nous nous bornerons à remarquer le fait que les deux modes d'association : latérale (biassociation paradesmique de Filipponi) ou bien du protomérite d'un individu avec le deutomérite de l'autre, à la manière de l'association entre satellites du genre Gregarina (biassociation prophypodesmique de Filipponi) se rencontrent.

D'après nous, ces deux modes d'association auraient le même but, c'est-à-dire la formation du kyste, ce dernier pouvant également probablement résulter d'une syzygie typique ( coppia gamontica » de Filipponi) observée par cet auteur chez les deux Stylocephalus de Blaps gigas.

Après la disparition de l'épimérite, les protomérites de $S$. variabilis de $A$. sericea ont un aspect un peu différent de ceux de la même espèce chez T. mucronata (fig. I, 5 et II, 1-2); mais cette différence ne nous a pas paru suffisante pour séparer les deux Grégarines. Elle est peut-être simplement due à la vie dans un Ténébrionide différent, ce qui a pu provoquer de légères variations dans une même espèce.

B. L'Hirmocystis que nous avons trouvé chez Asida sericea est différent de $H$. asidx de Léger (1896) ; en effet, chez $H$. asida, les sporadins sont près petits, et les associations ne dépassent pas $20 \mu$.

L'espèce parasite de $A$. sericea est une forme beaucoup plus grande, et l'association est faite de deux individus qui sont toujours de taille différente, aussi la nommerons-nous : 


\section{Hirmocystis inæqualis n. sp.}

Dans une association, l'individu le plus grand a, par exemple, $100 \mu$ de long, et le conjoint $60 \mu$. Ils montrent un protomérite. arrondi, et un noyau sphérique avec un nucléole et de gros grains de paraglycogène (fig. II, 6).

Hirmocystis inæquctiis est relativement rare; on ne le trcuve pas chez tous les individus de A. sericea.

C. Certains A. sericea provenant de la même localité que celles qui hébergeaient les deux Grégarines précédentes, ainsi que d'autres (adultes et larves), récoltés à Banyuls, en mars 1951, étaient parasités par Stylocephalus oblongatus Hamm., déjà signalé chez Asida grisea, mais pour qui $A$. sericea est un hôte nouveau.

\section{3. - AKIS PUNCTATA Thunb. (= BACAROZZO Schrank)}

Des exemplaires de cette espèce, récoltés en juin 1950 à Stretta di Patrimonio, côte Ouest de la base du Cap Corse (Corse), nous ont montré, dans leur intestin, une Grégarine appartenant au genre Sphærorhynchus.

Schneider (1886) a décrit chez Akis sp. d'Oran Sphærocephalus ophioïdes; Labbé (1899) change le nom de Sphærocephalus en celui de Sphærorhynchus, le premier étant préoccupé par un Coléoptère.

La Grégarine d'Akis punctata quoiqu'appartenant sûrement au même genre, nous a semblé différer de celle trouvée par Schneider chez les Akis africains. Nous en faisons donc une espèce nouvelle :

\section{Sphærorhynchus hamoni n. sp.}

dédiée à $\mathbf{J}$. Hamon qui a bien voulu récolter et nous envoyer les Akis de Corse.

Les petites formes de $S$. hamoni montrent un épimérite sphéruleux, prolongé antérieurement par une pointe qui s'enfonce dans l'épithélium intestinal de l'hôte. Un col assez court le relie au protomérite (fig. III, 1) ; le deutomérite ovoïde est de la même longueur que l'épimérite et le protomérite réunis ; il contient un noyau sphérique avec nucléole central et chromatine granuleuse.

Puis, la pointe antérieure de l'épimérite disparaît; celui-ci est alors fait d'une sphérule portéa par un col qui s'est allongé (fig. III, 2) ; la limite entre l'épimérite et le protomérite devient nette (fig. III, 3) ; le deutomérite s'accroit, s'allonge et s'effile à sa partie postérieure. Le noyau occupe toujours une position centrale ; 


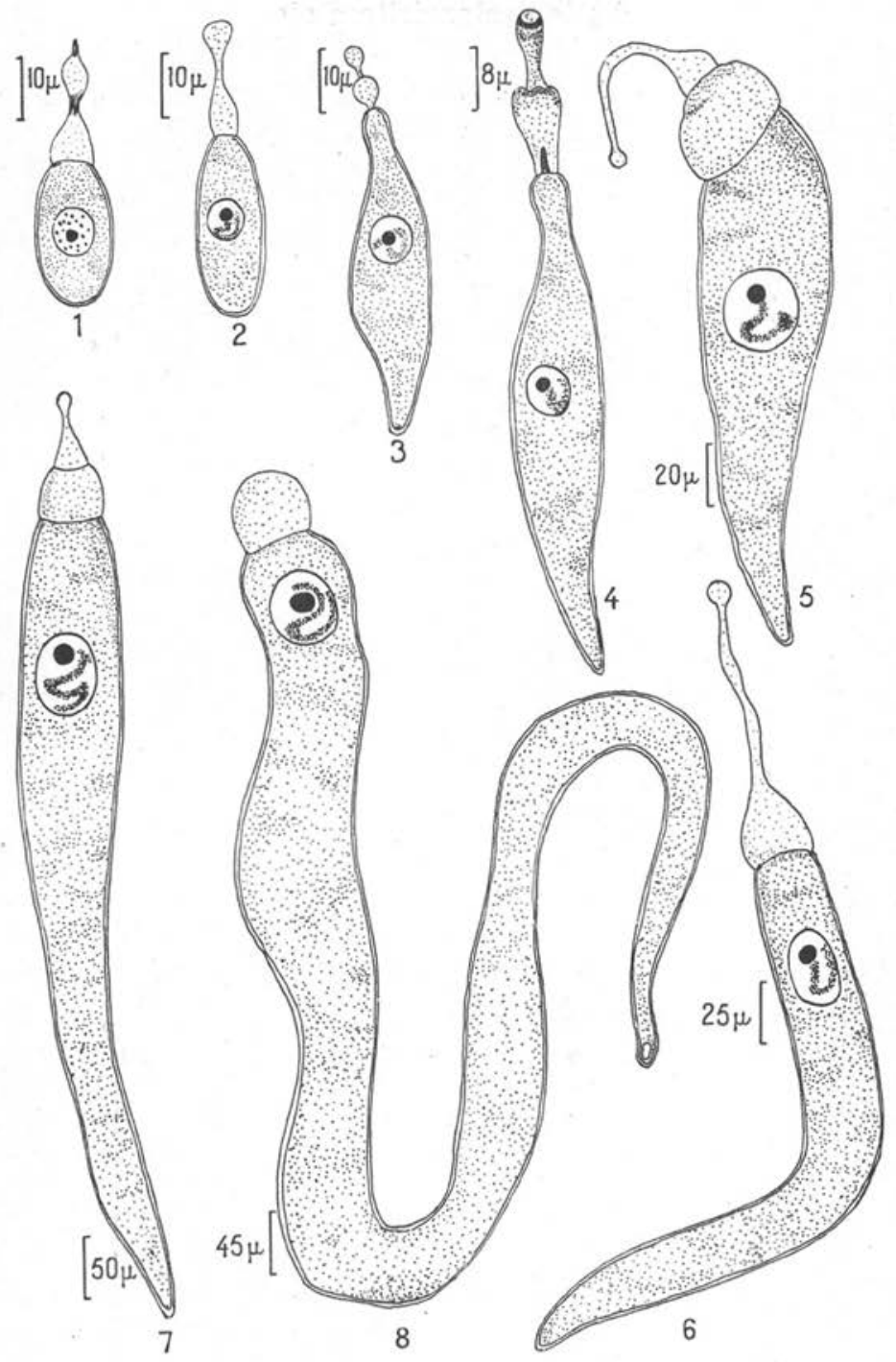

FIc. III. - Grégarine de Akis punctata Thunb.

1-2-3-4-5-6-7-8, divers stades de l'évolution de Spharorhynchus hamonı n. sp.

la chromatine s'est́ réunie en un filament contourné, dont une extrémité est accolée au nucléole (fig. III, 4): Pendant la croissance de la Grégarine, le col de l'épimérite continue à s'allonger ; il est toujours terminé par une sphérule ; l'ensemble de l'épimérite atteint 
$80 \mu$ de long (fig. III, 5) ; le protomérite a alors $26 \mu$ et le deutomérite $160 \mu$.

Le deutomérite poursuit sa croissance (fig. III, 6) ; le noyau montre toujours le nucléole et le cordon de chromatine qui lui est accolé par une de ses extrémités; dans le protoplasme, les petits grains de paraglycogène apparaissent plus nombreux, et la membrane externe s'épaissit.

Le Sphærorhynchus se détache alors de l'épithélium intestinal et devient libre dans la lumière pendant que l'épimérite régresse (fig. III, 7). Il ne se montrera plus que comme un petit bouton clair à la partie antérieure du protomérite qui lui-même finira par s'effacer.

Les sporadins atteignent une grande taille et sont très déformables (fig. III, 8). Nous en avons trouvé mesurant $1 \mathrm{~mm} .2$ de longucur. Le protomérite est ovoïde ou trapézoïdal. Le deutomérite très allongé contient antérieurement un noyau relativement petit qui ne dépasse pas $40 \mu$ de diamètre. La cuticule est épaisse, et l'extrémité postérieure claire montre une vacuole; le protoplasme est rempli de petits granules de paraglycogène.

$S$. hamoni que nous venons de décrire diffère de $S$. ophiödes, tout d'abord par la taille beaucoup plus petite des céphalins et sporadins; le céphalin de cette dernière espèce mesure $1 \mathrm{~mm} .3$ dont $200 \mu$ pour l'épimérite, celui de $S$. hamoni ne dépasse pas $800 \mu$ et l'épimérite $110 \mu$. Le sporadin de $S$. ophiö̈des atteint 3 à $4 \mathrm{~mm}$., alors que le plus grand de $S$. hamoni que nous ayons observé ne dépassait pas $1 \mathrm{~mm} .5$.

Les deux Sphærorhynchus diffèrent aussi par la forme de l'épimérite qui se termine dans les deux espèces par un bouton sphérique, mais $S$. hamoni a un épimérite en col, beaucoup plus effilé que celui de $S$. ophioüdes qui, d'après le dessin de Schneider, est massif. L'épimérite de $S$. hamoni par contre, ressemble à un épimérite de Stỵlocephalus.

Nous pensons qu'en réalité Sphærorhynchus et Stylocephalus sont deux genres très voisins qu'il faudrait peut-être réunir en un seul. Ce qui nous a, en tout cas, empêché de rattacher ici $S$. hamoni à Stylocephalus variabilis est le fait que les sporadins de cette dernière espèce ont toujours des formes plissées, et que le noyau de $S$. hamoni avec son ruban de chromatine attaché au nucléole par une de ses extrémités est différent de celui de Stylocephalus variabilis. 


\section{4. - AKIS ELEGANS Sol.}

Dans des individus appartenant à cette espèce, provenant de Casablanca (Maroc) et récoltés par le $\mathrm{D}^{\mathrm{r}} \mathrm{A}$. Chabaud, nous avons observé quelques stades d'une Grégarine qui doit appartenir au genre Sphærorhynchus, mais qui diffère de $S$. ophioides Schneider et de $S$. hamoni décrit ci-dessus ; elle est plus proche que cette dernière de la forme typique de Sphærorhynchus décrite par Schneider.

Les jeunes stades ont un épimérite à col court, terminé par quelques digitations protoplasmiques (fig. IV, 1 ) ; le deutomérite ovoïde a environ la longueur de l'épimérite et du protomérite réunis.

Le noyau, petit, a un gros nucléole ; l'épimérite n'est jamais muni d'un long col (fig. IV, 2) et finit par n'être plus représenté que par une sphérule avec substance spumeuse qui le fait adhérer à l'épithélium intestinal (fig. IV, 3). La membrane du deutomérite est épaisse et striée, et le
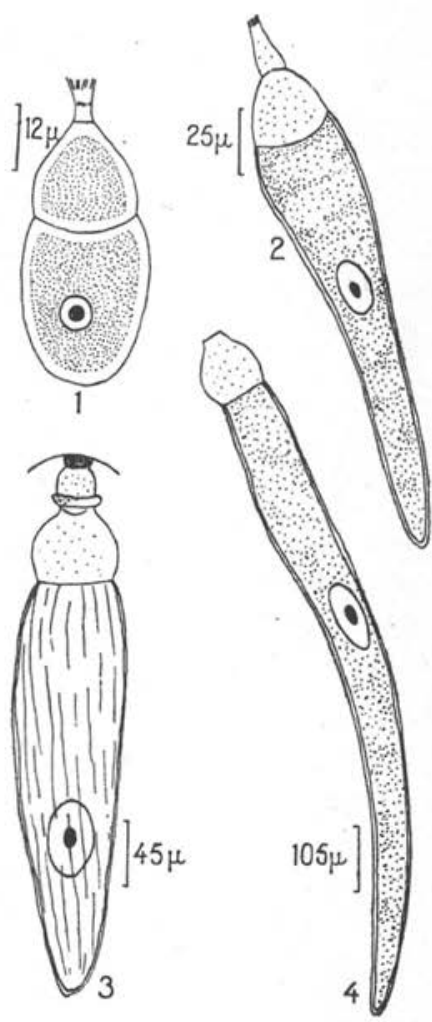

FIG. IV. - Grégarine de Akis elegans Sol. 1-2-3-4, Sphærorhynchus chabaudi n. sp. noyau postérieur.

Nous avons enfin observé de grandes formes (fig. IV, 4) mesurant $1.555 \mu$ de long, à protomérite trapézoïdal rappelant celui de $S$. $h a-$ inoni. Nous nommerons cette espèce :

\section{Sphærorhynchus chabaudi n. sp.}

la dédiant au $\mathrm{D}^{r} \mathrm{~A}$. Chabaud qui a bien voulu nous donner des Akis elegans de son élevage. 
5. - PIMELIA ANGULATA F. ssp. ANGULOSA Ol. (1)

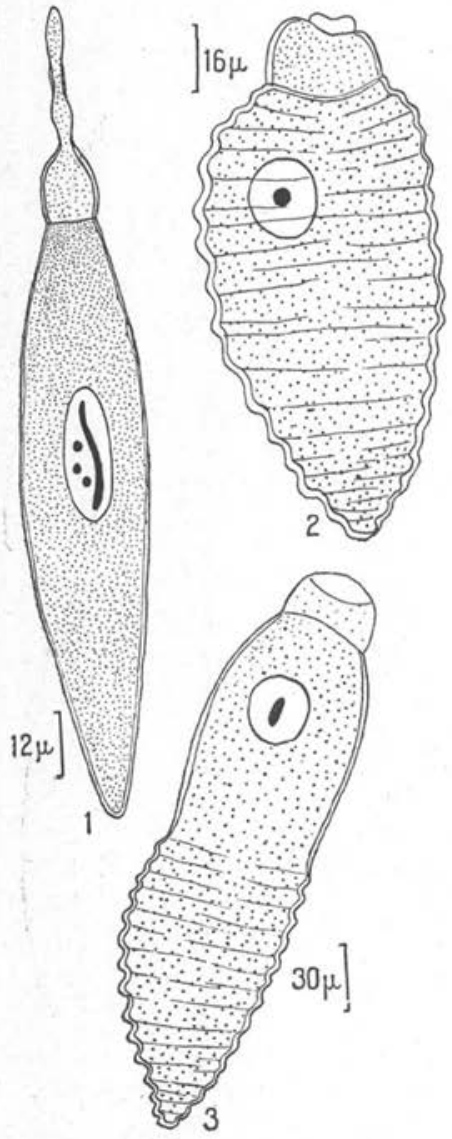

F1G. V. - Grégarine de Pimelia angustata F., ssp. angulosa Ol. 1-2-3, Cystocephalus algerianus var. mauritanicus nov.

Schneider (1889) a créé le genre Cystocephalus avec l'espèce C. algerianus pour des Grégarines de l'intestin de Pimelia sp. d'Oran.

Nous avons retrouvé des Cystocephalus dans des exemplaires de Pimelia angulata ssp. angulosa provenant d'Atar (Mauritanie) et que nous devons à l'obligeance de notre collègue A. Descarpentries, Directeur du Vivarium du Muséum de Paris.

Les jeunes stades qui sont lisses montrent un épimérite fait de deux parties dont l'antérieure, ovoïde, est rattachée par un col plus rétréci au protomérite. Le noyau montre plusieurs nucléoles et un ruban de chromatine (fig. V, 1). A côté de ces formes lisses qui, devenues libres dans la lumière intestinale par perte de l'épimérite peuvent atteindre $500 \mu$, s'observent des formes segmentées (fig. V, 2).

Celles-ci, généralement plus trapues, on $\mathrm{t}$ une membrane épaisse et plissée et un noyau sphérique.

Comme chez Stylocephalus variabilis existent des formes partiellement segmentées (fig. V, 3). Dans ce cas, c'est généralement la partie postérieure du deutomérite qui montre le plissement.

Ces formes de passage montrent bien que nous avons affaire à une même espèce ; les Pimelia de Mauritanie montrent une infestation intense et les formes lisses et plissées sont mélangées.

(1) Nous remercions ici notre collègue F. Español (Barcelone), qui a bien voulu nous déterminer ce Ténébrionide. 
Le protoplasme de ces Grégarines contient des grains de paraglycogène et de nombreux granules réfringents de pigment.

Nous conservons pour ces Cystocephalus le nom de C. algerianus donné par Schneider, mais nous en ferons la variété mauritanicus N. vaR.; pour concrétiser les différences qui existent entre les deux fo:mes et en particulier la fréquence des individus plissés.

\section{RÉSUMÉ ET CONCLUSIONS}

Les quelques espèces de Coléoptères Ténébrionides que nous avons examinées afin d'étudier les Grégarines Polycystidées qu'elles hélıergent nous ont donné les résultats suivants :

\begin{tabular}{|c|c|c|}
\hline CoLÉoptÈres HôTES & GRÉGARINES & FaMiLLes \\
\hline Tentyria mucronata Stev..... & $\begin{array}{l}\text { Stylocephalus variabilis n. sp. } \\
\text { Sphaerocystis tentyriae n. sp. }\end{array}$ & $\begin{array}{l}\text { Stylocephalidae } \\
\text { Gregarinidae. }\end{array}$ \\
\hline Asida sericea $01 . . . . \ldots \ldots \ldots . . .$. & $\begin{array}{l}\text { Stylocephalus variabilis n. sp. } \\
\text { Siylocephalus oblongatus } \\
\text { Hamm. } \\
\text { Hirmocystis inacqualis n. sp. }\end{array}$ & Gregarinide. \\
\hline Akis punctata Thunb.......... & $\begin{array}{l}\text { Sphaerorhynchus hamoni } \\
\text { n. sp. }\end{array}$ & Stylocephalidae. \\
\hline Akis elegans Sol............... & $\begin{array}{l}\text { Sphaerorhynchus chabaudi } \\
\text { n. sp. }\end{array}$ & Stylocephalidx. \\
\hline $\begin{array}{l}\text { Pimelia angulata F. ssp. an- } \\
\text { gulosa } 01 \ldots \ldots \ldots \ldots \ldots \ldots \ldots\end{array}$ & $\begin{array}{l}\text { Cystocephalusalgerianus Schn. } \\
\text { var. mauritanicus nov. }\end{array}$ & St!llocephalidae. \\
\hline
\end{tabular}

Ce tableau montre, une fois de plus, la fréquence des Stylocephalidar chez les Coléoptères Ténébrionides.

Ce qui semble caractériser ces Grégarines, c'est la présence, chez plusieurs espèces, de formes d'aspect segmenté à cuticule épaisse et plissée (1), mélangées à des formes lisses de la même espèce. Des

(1) Depuis la rédaction de ces lignes, nous avons vu un travail de Misra (1941) sur Stylocephalus indicus Misra, Grégarine trouvée chez le Ténébrionide Gonocephalum helopioides Frm. aux Indes. Cet auteur a remarqué chez cette espèce l'existence de formes annelées, qu'il interprète comme une réaction de la Gré- 
formes à la fois lisses et plissées font le passage entre ces deux extrêmes.

Nous avons déjà signalé la coexistence de ces deux formes chez Actinocephalus licini Tuz. et Théod., parasite de Licinus punctatulus Fab. (Tuzet et Théodoridès, 1951) ; nous les avons retrouvées ici chez Stylocephalus variabilis et Cystocephalus algerianus var. mauritanicus.

Ces Grégarines, d'aspect segmenté, fréquentes chez les Coléoptères, sont peut-être une réaction du parasite vis-à-vis du milieu intestinal de l'hôte qui est susceptible de présenter des propriétés particulières que l'un de nous (J.'T.) a l'intention d'étudier dans un avenir proche.

\section{BiBLIOGRAPHIE}

ElLis (M. M.). - A new species of Polycystid Gregarine from the United States. Zool. Anz., XXXIX, 1912, 25-27.

Filipponi (A.). - Studi sugli Stylocephalide (Sporozoa). I. Due nuovi Stilocefalidi parassiti di Blaps gigas L. Riv. di Parasitol., X, 1949, 4, 205-229, 2 planches.

Foersten (H.). - Gregarinen in Schlesischen Insekten. Z. f. Parasit., X, 1939, 157-209.

- Beobachtungen über das Auftreten von Gregarinen in Insekten. Ibid., 1939, 644-674.

LabBÉ (A.). - Sporozoa (in Das Tierreich, V, 1899, 180 p., 196 fig.).

LÉGer (L.). - Recherches sur les Grégarines. Tábl. Zool., III, 1892, 1-183.

- Nouvelles recherches sur les Polycystidées parasites des Arthropodes terrestres. Ann. Fac. Sci. Marseille. VI, 1896, 1-54.

Mrspa (P. L.). - Observations on a new Gregarine Stylocephalus bahli sp. nov. from the alimentary canal of an Indian beetle Gonocephalum helopioides. Rec. Ind. Mus., XLIII, 1941, 43-72, 1 pl.

Schnetder (A.). - Grégarines nouvelles ou peu connues. Tabl. Zool., I, 1886, 90-103.

TUzet (O.) et THÉodorıdès (J.). - Actinocephalus !icini n. sp. et Cometoüdes licini n. sp., nouvelles Grégarines parasites de l'intestiu de Licinus punctatulus Fab. Arch. Zool. Exp. Gén., LXXXVilI, 1951, Notes et Revue (sous presse).

WAT'sox (M. E.). - Studies on Gregarines. I. Illinois Biol. Monoggr., XI, 1916, 3, 258 p.

[Laboratoire de Zoologie de l'Université de Montpellier et Laboratoire Arago, de l'Université de Paris, Banyuls-sur-Mer (P.-O.)]

garine aux obstacles qu'clle heurte au cours de sa très lente progression. Nous avons cependant observé ces annulations chez des individus fixés immédiatement après la dissection de l'hôte et qui, par conséquent, n'avaient pas eu le temps de progresser sur la lame. L'explication de Misra nous parait peu vraisemblable car l'existence de formes annelées chez les Grégarines de Coléoptères semble assez fréquente et doit être due à d'autres causes. 